\title{
Single-Shot Spin Readout in Semiconductors Near the Shot-Noise Sensitivity Limit
}

\author{
D. Keith॰, M. G. House, M. B. Donnelly, T. F. Watson, B. Weber, and M. Y. Simmons \\ Centre of Excellence for Quantum Computation and Communication Technology, School of Physics, \\ University of New South Wales, Sydney, New South Wales 2052, Australia
}

(Received 9 May 2019; published 3 October 2019)

\begin{abstract}
Fault-tolerant quantum computation requires qubit measurements to be both high fidelity and fast to ensure that idling qubits do not generate more errors during the measurement of ancilla qubits than can be corrected. Towards this goal, we demonstrate single-shot readout of semiconductor spin qubits with $97 \%$ fidelity in $1.5 \mu \mathrm{s}$. In particular, we show that we can engineer donor-based single-electron transistors (SETs) in silicon with atomic precision to measure single spins much faster than the spin decoherence times in isotopically purified silicon ( $270 \mu \mathrm{s})$. By designing the SET to have a large capacitive coupling between the SET and target charge, we can optimally operate in the "strong-response" regime to ensure maximal signal contrast. We demonstrate single-charge detection with a signal-to-noise ratio (SNR) of 12.7 at $10 \mathrm{MHz}$ bandwidth, corresponding to a SET charge sensitivity (integration time for SNR =2) of $2.5 \mathrm{~ns}$. We present a theory of the shot-noise sensitivity limit for the strong-response regime which predicts that the present sensitivity is about one order of magnitude above the shot-noise limit. By reducing cold amplification noise to reach the shot-noise limit, it should be theoretically possible to achieve high-fidelity, single-shot readout of an electron spin in silicon with a total readout time of approximately $36 \mathrm{~ns}$.

DOI: 10.1103/PhysRevX.9.041003

Subject Areas: Quantum Information,

Semiconductor Physics
\end{abstract}

Sensitive detectors capable of fast, high-fidelity, singleshot measurements of quantum states are essential for robust quantum computation [1,2]. Single-electron spins in semiconductor devices are attractive for quantum computation as they have a high predicted ratio of coherence times to gate operation times, $\sim 10^{5}-10^{8}$ [3-7]. However, for fault tolerance, fast readout times are also essential to ensure the accurate measurement of multiple qubits before idling qubits decohere [8,9]. Readout times faster than decoherence rates can also unlock the possibility for new experiments such as entanglement via measurement and quantum steering [10,11]. To date however, the fastest electron single-spin state measurements in semiconductor devices have taken $>100 \mu \mathrm{s}[12,13]$, comparable to the best decoherence times $(270 \mu \mathrm{s})$ in isotopically purified silicon [14-16]. Here, we demonstrate high-fidelity (97\%) single-shot readout of electron spins in silicon in $1.5 \mu \mathrm{s}$, nearly 2 orders of magnitude faster than previous results. Critical to the result is the nanoscale engineering

\footnotetext{
*Present address: School of Physical and Mathematical Sciences, Nanyang Technological University, 637371 Singapore. †michelle.simmons@unsw.edu.au

Published by the American Physical Society under the terms of the Creative Commons Attribution 4.0 International license. Further distribution of this work must maintain attribution to the author(s) and the published article's title, journal citation, and DOI.
}

of single-electron transistor (SET) detectors to reach the strong-response regime [17]. Our nanoscale detectors are designed simultaneously both for large charging energies $\left(E_{C} \approx 4 \mathrm{meV}\right)$ to achieve true Coulomb blockade and for highly conductive tunnel barriers for maximum signal contrast. With such a detector we demonstrate singlecharge detection with a signal-to-noise ratio (SNR) of 12.7 with $10 \mathrm{MHz}$ bandwidth. As opposed to the shot-noise sensitivity limit for SETs in the weak-response regime [18-22], here we present the sensitivity in the strongresponse regime, which is more suitable for binary spin detection, and show that our results are within one order of magnitude of the shot-noise limit.

In Fig. 1(a) we show a scanning tunneling microscope (STM) image of a nanoengineered single-electron transistor used for fast, high-fidelity spin and charge state readout. The device consists of two phosphorus donor quantum dots ( $D 1=3 \mathrm{P}$ and $D 2=2 \mathrm{P}$ ) tunnel coupled (18 nm away) to a large donor island (SET) with source $(S)$ and drain $(D)$ leads that form a SET detector [23]. The detector is $\sim 100 \mathrm{~nm}^{2}$ in size, consisting of $\sim 100 \mathrm{P}$ donor atoms, and has been designed with tunnel barriers of $\sim 10 \times 5 \mathrm{~nm}^{2}$ for both source and drain leads to enable a high conductance of $\sim 10 \mu \mathrm{S}$. The SET is connected via an impedance matching $L C$ tank circuit to a rf reflectometry homodyne detection setup, as shown in Fig. 1(a), to measure the radio-frequency response of the SET (rf SET) [24]. The inductance $L_{0}=1200 \mathrm{nH}$ combined with the parasitic 
capacitance $C_{p}=0.4 \mathrm{pF}$ is chosen to ensure the rf SET is impedance matched with the $50 \Omega$ transmission lines for maximal signal contrast (see the Appendix A). An input carrier wave, with frequency $f=223 \mathrm{MHz}$, is reflected by the $L C$ circuit depending on the SET conductivity, and the real $(I)$ and imaginary $(Q)$ components of the reflected signal are measured.

We show the strong response of the SET to charge movement in the device, while measured in a dilution refrigerator with $60 \mathrm{mK}$ base temperature, as a function of gate voltages in Fig. 1(b). Here we observe a large shift $(\sim 20 \mathrm{mV})$ in the $\mathrm{rf}$ response, 4 times the $5 \mathrm{mV}$ Coulomb peak width, as a single electron is loaded onto the left donor dot. The ratio we measure is comparable to results in other donor-based systems [12,13], in comparison to quantum dot systems [25] where the ratio is often less than one. This large shift arises from the strong coupling between the SET and donor quantum dot due to the large mutual charging energy $(\Delta \mu \sim 1.3 \mathrm{meV})$ between them. The large relative
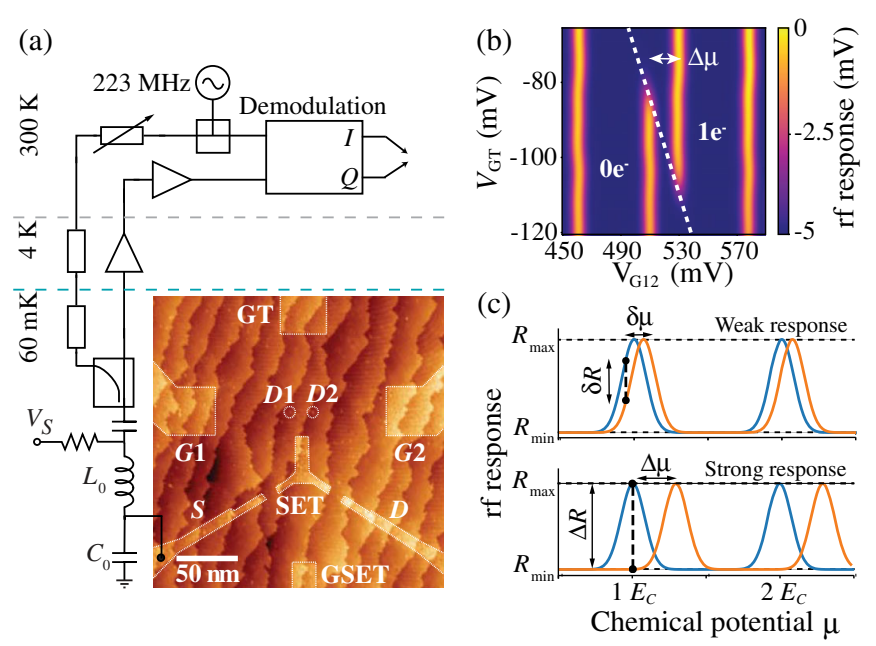

FIG. 1. Strong response of a donor-based rf SET. (a) STM image of the device with external reflectometry circuit schematic. Lighter patches are regions where the passivating $\mathrm{H}$ layer has been removed ready for $\mathrm{P}$ incorporation to create in-plane conducting gates ( $G 1, G 2$, GT, and GSET), SET charge detector (SET, $S$, and $D$ ), and donor quantum dots ( $D 1$ and $D 2$ ). A radiofrequency carrier wave is attenuated down to the $60 \mathrm{mK}$ stage where it is reflected by the resonant $L C$ tank circuit, amplified at $4 \mathrm{~K}$ and room temperature, and then demodulated into $I$ and $Q$ components. (b) Charge stability diagram showing the rf SET response as a function of gate voltages $V_{\mathrm{GT}}$ and $V_{G 12}$ (linear combination of $V_{G 1}$ and $V_{G 2}$ ) at an electron transition on $D 1$. (c) The contrast in rf response $(\delta R, \Delta R)$ of the SET as a function of the chemical potential $\mu$ in the weak-response (upper) and strong-response (lower) regimes, respectively, in terms of the SET charging energy $E_{C}$. The $\mathrm{rf}$ response reaches $R_{\min }$ during Coulomb blockade and $R_{\max }$ atop a Coulomb peak and shifts by the mutual charging energy $(\delta \mu, \Delta \mu)$ in the respective weakresponse and strong-response regimes due to a single-electron transition. The SET signal can switch between $R_{0}$ (lower black dot) and $R_{1}$ (upper black dot) for a given chemical potential. shift in our SET detector places it well within the "strongresponse" regime, in which the difference $\Delta R$ in the SET signal levels of the two charge states, $R_{0}$ and $R_{1}$ [black dots in Fig. 1(c)], is approximately equal to the difference between the minimum $R_{\min }$ (Coulomb blockade) and maximum $R_{\max }$ (Coulomb peak) SET signal, as illustrated in the lower panel of Fig. 1(c) [17]. Here, the detection sensitivity of the SET is fundamentally limited by shot noise, the noise that results from the stochastic nature of individual electron tunneling events through the SET [17]. The strong-response regime distinguishes our experiment from previous rf SET experiments aimed at achieving shotnoise limited sensitivity, which have traditionally been operated as weak-response sensors [21,22,24]. Indeed, most theoretical studies on the shot-noise sensitivity limit have assumed a weakly responding SET, in which a small SET response $\delta R$ results from a small shift in its potential $\delta \mu$ [Fig 1(c), upper panel] [18-20]. As a consequence, the sensitivity metric is typically expressed in units of $e / \sqrt{\mathrm{Hz}}$, emphasizing the smallest detectable potential shift. However, this metric does not address the single-charge detection sensitivity, a key factor in the overall determination of the spin readout fidelity, which also depends on the size of the capacitive coupling between qubit and sensor, and has a binary outcome. A better metric for binary charge detection sensitivity is the "measurement time" $\tau_{m}$, as adopted in superconducting qubits, which uses the integration time required to resolve a charge with a signal-to-noise ratio (SNR) of 2 [26,27]. To clarify the key differences between these two regimes, consider that experimentally, to achieve shot-noise limited sensitivity in the weak-response regime, the device is tuned to the side of a Coulomb peak where the slope $\delta R / \delta \mu$ is maximal, and the rf power is limited to avoid broadening the peak and reducing the slope. For a strong-response SET, however, we tune the SET such that its conductance is maximal for one charge state and zero for the other, so that we can apply more rf power before local heating starts to limit the sensitivity. Because of the differences in these experimental approaches, it is not possible to compare sensitivities from one experiment to another. Instead, by expressing the shot-noise limit in terms of the measurement time as used in superconducting qubits, we find charge sensitivities, $\tau_{m}=2.5 \mathrm{~ns}$, approximately one order of magnitude above the limit and faster than other semiconductor spin measurement experiments, which have demonstrated $\tau_{m}=50-1000 \mathrm{~ns}$ to date $[12,13,28-30]$.

The ability to engineer donor-based devices at the nanoscale using atomic precision lithography [31] has multiple advantages for creating a SET capable of reaching the strong-response regime. The capacitive coupling between the SET and donor dots is particularly strong in STM donor devices due to their small separations $(\sim 20 \mathrm{~nm})$ in the same crystallographic plane. The donors' Coulomb potential defines the conducting regions of the 
device so that no extra gates are required for confinement, ensuring minimal shielding of the capacitive coupling. The small SET island $(\sim 50 \mathrm{~nm})$ produces a large charging energy $E_{C}$ that separates each of the Coulomb peaks with a region of true blockade where the SET conductance is zero. The sharp and deep confinement of the donors' Coulomb potential also ensures that we are independently able to engineer small SET tunnel gaps $\left(\sim 10 \times 5 \mathrm{~nm}^{2}\right)$ with high conductance to maximize the SET's absolute response contrast. Combined, these four attributes of precision atomic qubit engineering, the large charging energy of a nanometer SET island, the small, highly conductive tunnel barriers, the small separations of components in the same plane, and the minimal gate density with minimal shielding to allow large mutual charging energies, result in a highly sensitive charge and spin detector.

To demonstrate high-speed $(\mu \mathrm{s})$ charge detection, we show real-time traces sampled at $10 \mathrm{MHz}$, in Fig. 2(a). Here, we tune the SET and $D 2$ donor dot potentials so that single electrons tunnel between them, and show that the rf SET is sensitive enough to detect the single-electron tunneling events on the sub- $\mu$ s timescale. The $51 \mathrm{~ns}$ response time of our detector is limited by the resonance of the $L C$ circuit with an effective quality factor varying from 20 to 50 and carrier wave frequency $f=223 \mathrm{MHz}$
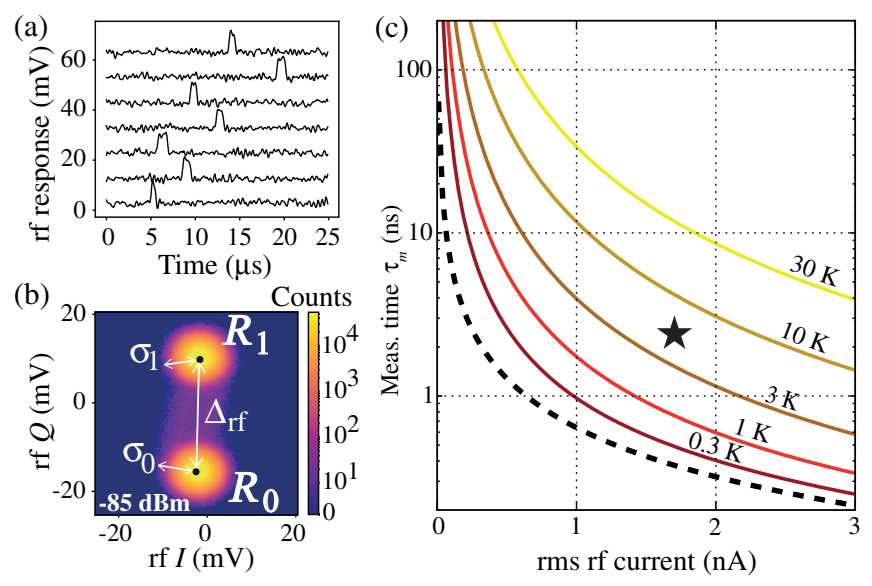

FIG. 2. High-sensitivity charge detection. (a) Examples of charge transition events of the third electron on the right donor dot (D2) detected by the change in rf SET response. (b) 2D histogram in $I-Q$ space showing the bimodal Gaussian distribution of many charge detection traces of the third electron on $D 2$ measured with $-85 \mathrm{dBm}$ rf power reaching the device. The distribution shows two clear peaks centered around $R_{0}$ and $R_{1}$ (separated by $\Delta_{\mathrm{rf}}$ ) with standard deviations of noise $\sigma_{0}$ and $\sigma_{1}$ corresponding to the respective $\mathrm{ON}$ and $\mathrm{OFF}$ electron charge states on the donor quantum dot. (c) The theoretically optimal sensitivity, characterized by the measurement time $\tau_{m}$, as a function of the rms rf current through the rf SET. The black dashed line indicates the theoretical shot-noise limit. Solid curves show how $\tau_{m}$ increases with additional amplifier noise, with noise temperature between 0.3 and $30 \mathrm{~K}$. A star indicates approximately the sensitivity achieved in the present experiment. (see Appendix B for characterization of the circuit response times). Many such traces are used to create a histogram of the $I$ and $Q$ rf-response components [Fig. 2(b)]. The histogram shows two distinct regions corresponding to the "ON" ( 3 electrons) and "OFF" ( 2 electrons) charge states of the third electron on the right donor $\operatorname{dot}(D 2)$. These regions are then characterized by their respective means $\left(R_{0}\right.$ and $R_{1}$ separated by $\left.\Delta_{\mathrm{rf}}\right)$ and standard deviations $\left(\sigma_{0}\right.$ and $\left.\sigma_{1}\right)$. The SNR, defined as SNR $=\Delta_{\mathrm{rf}} / \sqrt{\frac{1}{2}\left(\sigma_{0}^{2}+\sigma_{1}^{2}\right)}$, is optimal when we input $-85 \mathrm{dBm}$ power to the device, with a compromise between the high reflected signal contrast and Coulomb peak broadening, resulting in SNR $=12.7$ with a $10 \mathrm{MHz}$ filter bandwidth. The results correspond to a short measurement time, $\tau_{m}=2.5 \mathrm{~ns}$; see the black star in Fig. 2(c). For clarification, the measurement time is the integration time required to distinguish between charge states. The time required to distinguish between spin states, the readout time, is inherently longer based on the detector's ability to respond to changes in states, i.e., response time, and the characteristic tunnel rate of the electron between the qubit and SET island.

The SET sensitivity quantifies how well we can distinguish between two charge states, which correspond to SET rms currents $I_{0}$ and $I_{1}$, respectively. We can model the ideal case where the current through the SET is zero for one of the charge states (i.e., $I_{0}=0$ ), such that the measurement time would be dictated by the time taken for a single electron to transit the SET, i.e., $\tau_{m} \sim e / I_{1}$ [27]. In other words, as soon as a single electron tunnels in this case, we determine we are in the charge state corresponding to $I_{1}$. We can calculate the sensitivity in this limit by assuming the SET, with impedance $Z_{\mathrm{SET}}$, is coupled to a noiseless amplifier of input impedance $Z_{0}$ through a perfect impedance matching circuit. The current delivered to the amplifier is $I_{A}=I_{1} \sqrt{Z_{S E T} / Z_{0}}$ and the shot noise is approximately $\sigma_{A}^{2} \approx 2 e F I_{1}\left(Z_{\mathrm{SET}} / Z_{0}\right) / \tau$, where $F$ is the Fano factor and $\tau$ is the integration time (see Appendix $\mathrm{C}$ for analysis of the device shot noise). The measurement time for $Z_{\mathrm{SET}}=100 \mathrm{k} \Omega, \tau_{m}$, is defined as the integration time such that the signal-to-noise ratio $I_{A} / \sigma_{A}=2$ is plotted as the black dashed line in Fig. 2(c). Colored curves in Fig. 2(c) illustrate how the $\tau_{m}$ increases with finite noise temperature of the preamplifier. The black star illustrates approximately the sensitivity achieved in this experiment after taking losses into account (see Appendix D for details on these calculations). This graph shows that for realistic currents through the SET ( 1 nA) the shot-noise limit of $\tau_{m}$ can reach below 200 ps. Indeed, our theoretical calculations show that with shot-noise limited sensitivity, and an optimal tunnel rate of approximately $5.4 \mathrm{~ns}$, a single-spin measurement can be completed with error rates $<1 \%$ in $\sim 36 \mathrm{~ns}$, more than 5000 times faster than ${ }^{28} \mathrm{Si}$ decoherence processes [32]. Taking current reflectometry methods into consideration, a more practical readout time 
would be on the order of 400 ns (see Appendix E for further discussion).

We now consider high-speed single-shot spin readout at the third electron transition of the left donor quantum dot $D 1$ [real-time traces shown in Fig. 3(a)]. This particular electron transition has a characteristic tunnel rate $\sim 1 \mathrm{MHz}$ and had been inaccessible to previous dc measurements due to its fast tunnel rate [23]. The tunnel events are now easily distinguished with the rf SET due to the high SNR $=8.1$ (sensitivity $\tau_{m}=3 \mathrm{~ns}$ ) with $10 \mathrm{MHz}$ filter bandwidth and $50 \mathrm{~ns}$ integration time. The fidelities $F^{\uparrow}$ and $F^{\downarrow}$ are used to quantify the percentage of correctly identified spin-up $(|\uparrow\rangle)$ and spin-down $(|\downarrow\rangle)$ electrons, respectively. A series of tunnel rate equations [Eqs. (F4) and (F5) in Appendix F] are used to optimize the visibility $V=F^{\uparrow}+F^{\downarrow}-1$ with respect to $\Delta t$, and the complement of the visibility and fidelities are shown in Fig. 3(b). For short $\Delta t, V$ is limited by $F^{\uparrow}$ as there is not enough time to detect $|\uparrow\rangle$ electrons tunneling off the donor dot. For long $\Delta t, V$ is limited by $F^{\downarrow}$ because thermal excitations of $|\downarrow\rangle$ electrons start to dominate and are misidentified as $|\uparrow\rangle$ tunnel events. The readout position, relative to where the SET and donor dot's potentials are equal, was also chosen to be low enough to minimize unintentional tunneling events caused by heating effects while also ensuring that tunnel events are long
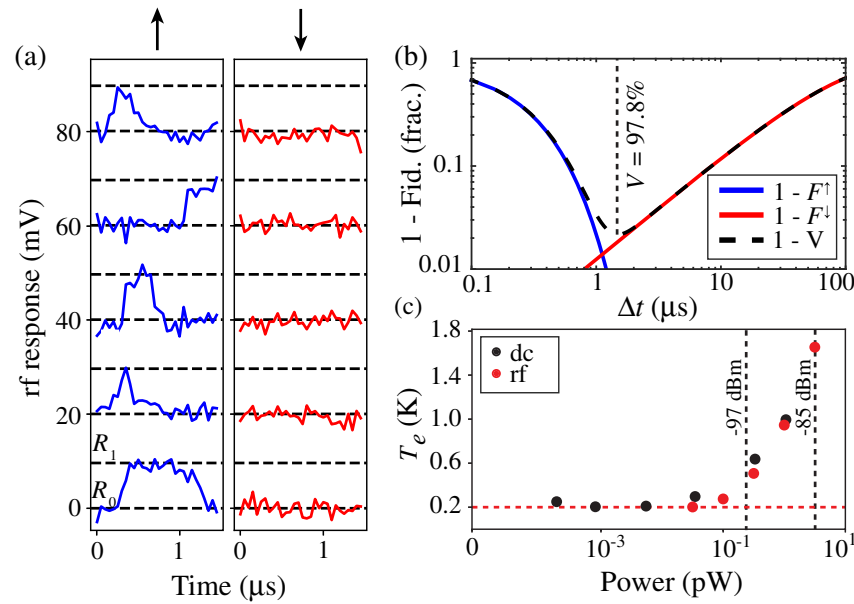

FIG. 3. $\mu$ s electron spin readout. (a) Single-shot spin readout is performed in a global magnetic field $B=1.5 \mathrm{~T}$ by pulsing gate voltages to read spin states via energy selective tunneling. Each readout trace is declared spin-up or -down, respectively, if the detector response rises above a set threshold (blue, spin-up) or not (red, spin-down) during the optimal readout time $\Delta t_{\mathrm{opt}}=1.5 \mu \mathrm{s}$. Traces are offset for clarity with pairs of dotted lines depicting $R_{0}$ and $R_{1}$. (b) The readout time $\Delta t$ is varied to optimize state fidelities ( $F^{\uparrow}$ and $F^{\downarrow}$ for $|\uparrow\rangle$ and $|\downarrow\rangle$, respectively) and visibility $(V)$ and is optimal at $1.5 \mu \mathrm{s}$, where $V=97.8 \%$. (c) Increasing the rf-input power increases $T_{e}$ above $200 \mathrm{mK}$. When scaled to absolute applied power, heating due to dc current (black dots) and rf-input power (red dots) shows the same trend. Black dotted lines mark the optimal powers used for charge detection $(-85 \mathrm{dBm})$ and spin readout $(-97 \mathrm{dBm})$. compared to the charge detector's response time (51 ns) [13]. The optimal readout time $\Delta t=1.5 \mu \mathrm{s}(V=97.8 \%)$ is limited by the stochastic nature of the readout process and is 3 orders of magnitude faster than previous single-shot dc measurements of the same device [23]. The overall measurement fidelity $F_{M}$, when including electrical detection errors (see Appendix F for full determination of fidelities) is $97 \%$. The dominating factor that limits our readout fidelity is the response time of the resonant circuit which is not fast enough to detect a small, yet significant, number of faster tunneling events.

The reliance on stochastic energy-selective tunneling [33] makes single-electron spin readout more restrictive than charge detection, both thermally and temporally. To ensure high fidelities, the thermal energy $k_{B} T_{e}$ must be much less than the Zeeman splitting of the spin states $g \mu_{B} B$, where $T_{e}$ is the electron temperature, $k_{B}$ is Boltzman's constant, $g$ is the electron $g$ factor, and $\mu_{B}$ is the Bohr magneton. To minimize dissipation of energy during spin readout, and hence thermal excitations, we operate the $\mathrm{rf}$ SET with a rf-input power $12 \mathrm{dBm}$ lower than for charge detection, which leads to the reduced SNR. We observe that dissipation of energy due to the Joule heating of the SET results in an increase in the apparent local electron temperature above the $200 \mathrm{mK}$ baseline for powers $>10^{-1} \mathrm{pW}$, as shown in Fig. 3(c). For dc measurements, the SET bias $V_{\text {SET }}$ was varied, while for rf measurements, the rf-input power reaching the device was varied. The heating effect was the same whether the SET current was ac or dc, confirming that the observed heating is not directly caused by the $\mathrm{rf}$ excitation. The electron relaxation time $T_{1}$ is similarly unaffected as the reflectometry measurements replicated the dc results of $T_{1} \sim 20 \mathrm{~s}$ previously measured with the exact same device [23]. Temporally, the spin readout time $\Delta t$ must be longer than the integration time required for charge detection to take into account the time required for the stochastic tunnel events to occur. Tunnel events are then distinguished by comparing the maximum SET response within $\Delta t$ to a chosen threshold value; i.e., if at any point during $\Delta t$ the response rises above the threshold, the electron spin is declared $|\uparrow\rangle$, otherwise it is declared $|\downarrow\rangle[12]$.

The best spin readout performs measurements as fast as possible to complete experiments in reasonable time with good statistics, and much faster than decoherence times to minimize errors. In our single-electron spin system in silicon we have demonstrated fast $(1.5 \mu \mathrm{s})$ readout with high fidelity. The measurement speed is high enough to perform $\sim 500000$ measurements per second and is $\sim 180$ times faster than $T_{2}^{*}$ times ( $\left.270 \mu \mathrm{s}\right)$ measured in isotopically purified ${ }^{28} \mathrm{Si}$ [16]. The measurement rate compared to $T_{2}^{*}$ corresponds to decoherence errors $<0.4 \%$, as opposed to the $>30 \%$ that would correspond to previous measurement times $(100 \mu \mathrm{s}-100 \mathrm{~ms})$ in donor-based $\mathrm{Si}$ devices $[12,13,23,34]$. Currently there is much interest in 
gate-based, dispersive readout for semiconductor spin qubits, which reduces the number of leads in the device and may improve the prospects for scalability. Our experiment highlights the advantages of the SET for spin measurements: we have demonstrated an order of magnitude better sensitivity than the best dispersive readout sensitivity, even though that experiment used a low-noise Josephson parametric amplifier [35]. Furthermore the rf SET requires a matching circuit with only a quality factor $\approx 40$, whereas high-sensitivity dispersive readout uses $Q>1000$, giving the rf SET an advantage in overall bandwidth. Long term, for donor-based qubits, the required additional leads for the SET are proposed to reside in a three-dimensional architecture $[9,36]$, thereby providing a more scalable layout.

Currently, the fidelity of our fastest spin readout measurements is limited by the response time of our resonant tank circuit, which can be improved by designing it to operate at a higher carrier frequency (and could be improved by lowering the electron temperature $T_{e}$ ). The fidelity can be sufficiently high for error correction (>99\%) as long as the sample rate and SNR are suitably high [32], as has been achieved previously in similar experiments [23] as to that presented here, and could be further improved by adopting more sophisticated postprocessing techniques such as maximum likelihood methods [37]. The sensitivity can be improved in future experiments by reducing losses in the matching circuit and adopting an amplifier with lower noise temperature. In combination, it may be possible to achieve an order of magnitude improvement in charge detection sensitivity and readout of a singleelectron spin in approximately $36 \mathrm{~ns}$ with over 99\% measurement fidelity using such a detector in the strongresponse regime. Further improvements in the device itself, such as having higher SET conductance or tolerating more SET current without excessive heating, may see this time reduced further.

\section{ACKNOWLEDGMENTS}

The research was conducted by the Australian Research Council Centre of Excellence for Quantum Computation and Communication Technology (Project No. CE170100012) and Silicon Quantum Computing Pty Ltd. M. Y. S. acknowledges an Australian Research Council Laureate Fellowship.

T.F.W. and B.W. fabricated the device. D. K. and M. G. H. performed all the measurements. D. K., M. G. H., and M. B.D. analyzed all the data. The manuscript was written by D. K., M. G. H., M. B. D., and M. Y. S with input from all other authors. M. Y.S. supervised the project.

\section{APPENDIX A: IMPEDANCE MATCHING FOR THE STRONG-RESPONSE REGIME}

The impedance of a SET is necessarily high $(>10 \mathrm{k} \Omega)$, particularly compared with the characteristic impedance $Z_{0}$ of a laboratory coaxial cable (typically $50 \Omega$ ), so an

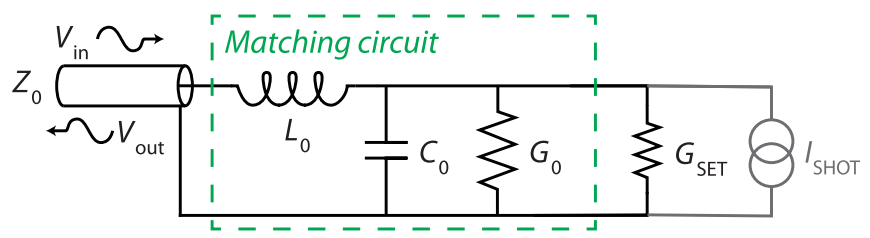

FIG. 4. rf SET matching circuit schematic. We represent the SET by conductance $G_{\mathrm{SET}}$, which is connected to a transmission line of characteristic impedance $Z_{0}$ via an $L$-match filter circuit consisting of inductance $L_{0}$, capacitance $C_{0}$, and parasitic dissipation represented by conductance $G_{0}$. The current source at right represents the shot-noise contribution of the SET, $I_{\mathrm{SHOT}}$.

impedance matching circuit is required to efficiently couple a rf signal to the SET. In this Appendix we consider the suitable design of an impedance matching circuit for a reflection measurement in which the input and output signals $V_{\text {in }}$ and $V_{\text {out }}$ interact with the circuit through a common port as shown in the left-hand side of Fig. 4. The reflected signal is related to the input signal by the reflection coefficient,

$$
\Gamma \equiv \frac{V_{\text {out }}}{V_{\text {in }}}=\frac{Z_{\text {in }}-Z_{0}}{Z_{\text {in }}+Z_{0}},
$$

where $Z_{\text {in }}$ is the total input impedance of the circuit [38]. For the purpose of detecting changes between two discrete charge states, the impedance matching circuit should be designed to maximize the contrast in the outgoing signal $\Delta V_{\text {out }}=\left|V_{\text {out }}\left(G_{1}\right)-V_{\text {out }}\left(G_{2}\right)\right|$ for two different SET conductance levels $G_{1}$ and $G_{2}$. We can impedance match our circuit by choosing values for the inductance $L_{0}$ and capacitance $C_{0}$ in our matching circuit to determine its characteristic impedance, $Z_{C}=\sqrt{L_{0} / C_{0}}$. We will next outline the best conditions to aim for when attempting to impedance match.

To determine how to achieve impedance matching practically, we model our matching circuit as shown in Fig. 4, consisting of an inductance $L_{0}$ in parallel with capacitance $C_{0}$ and having parasitic losses which we represent by a conductance in parallel with the capacitor, $G_{0}$. The circuit is connected to the SET, which has (variable) conductance $G_{\mathrm{SET}}$. The total input impedance of the circuit is

$$
Z_{\text {in }}(\omega)=i \omega L_{0}+\left(i \omega C_{0}+G\right)^{-1},
$$

where $G=G_{\mathrm{SET}}+G_{0}$. The resonant frequency of this circuit (at which the imaginary part of $Z_{\text {in }}$ is zero) is $\omega_{0}=\left(L_{0} C_{0}\right)^{-1 / 2}\left(1-Z_{C}^{2} G^{2}\right)$. For $\omega \approx \omega_{0}$,

$$
Z_{\text {in }}(\omega) \approx Z_{C}\left(\frac{1}{Q_{\text {int }}}+2 i \frac{\omega-\omega_{0}}{\omega_{0}}\right),
$$

where $Q_{\text {int }}=1 /\left(Z_{C} G\right)$ is the internal quality factor of the circuit. $Q_{\text {int }}$, together with the external quality factor, 
$Q_{\text {ext }}=Z_{C} / Z_{0}$, determines the total quality factor $Q=$ $\left(Q_{\text {int }}^{-1}+Q_{\text {ext }}^{-1}\right)^{-1}=Z_{C} /\left(Z_{0}+Z_{C}^{2} G\right)$. Although we are specifically considering the circuit in Fig. 4, Eq. (A3) is general to other resonant impedance transformers such as a quarterwave stub [39], or waveguide resonator [40]. Ignoring the small dependence of $\omega_{0}$ on $G_{\mathrm{SET}}$, we assume the circuit will be driven at the resonant frequency, in which case $Z_{\text {in }} \approx Z_{C}^{2} G=Z_{C} / Q_{\text {int }}$.

In practice, the input signal amplitude $V_{\text {in }}$ must be chosen to limit the voltage across the SET, $V_{\mathrm{SET}}$. Because $V_{\mathrm{SET}} \approx-i\left(Z_{C} G\right)^{-1} V_{\text {in }}$ is also a function of $Z_{C}$, we should consider the output signal contrast not in terms of $V_{\text {in }}$ but in terms of a fixed $\left|V_{\mathrm{SET}}\right|$. Practically, while we can adjust $V_{\text {in }}$ in an experiment, it is $\left|V_{\mathrm{SET}}\right|$ which determines the performance of the SET. Taking $G_{1}<G_{2}$, so that $\left|V_{\mathrm{SET}}\right|$ is larger when the SET conductance is $G_{1}$, experimentally we will choose $V_{\text {in }}$ so as to limit $\left|V_{\mathrm{SET}}\right|$. In that case,

$$
\left|\Delta V_{\text {out }}\right| \approx \frac{Z_{0} Z_{C}\left(G_{2}-G_{1}\right)}{Z_{0}+Z_{C}^{2}\left(G_{2}+G_{0}\right)}\left|V_{\mathrm{SET}}\right| .
$$

This is maximized for $Z_{C}=\sqrt{Z_{0} /\left(G_{2}+G_{0}\right)}$, which is the condition for impedance matching to SET conductance $G_{2}$ and leads to the optimized contrast

$$
\left|\Delta V_{\text {out }}\right|=\frac{\sqrt{Z_{0}}}{2 \sqrt{G_{2}+G_{0}}}\left(G_{2}-G_{1}\right)\left|V_{\mathrm{SET}}\right|
$$

Thus we have the result that to maximize the reflected signal amplitude the matching circuit should be designed to match the larger of the two total conductances (with $G_{0}$ ideally zero). The optimized contrast is independent of resonant frequency. For this particular experiment, in order to match the circuit for the larger conductance, we chose to minimize $C_{0}$ by relying on the parasitic capacitance of the device $C_{p}=0.4 \mathrm{pF}$ and chose the inductance to be $L_{0}=1.2 \mu \mathrm{H}$.

\section{APPENDIX B: MEASURED CIRCUIT BANDWIDTH}

An important consideration of the matching circuit is its bandwidth, or, equivalently, response time (not to be confused with the measurement time sensitivity metric):

$$
T=\frac{2 Q}{\omega_{0}}=\frac{2}{\omega_{0}} \frac{Z_{C}}{\left(Z_{0}+Z_{C}^{2} G\right)} .
$$

The output signal response to an instantaneous change in the SET conductance to $G$ will have a time dependence $V_{\text {out }} \propto \exp (-t / T)$. The response time for the impedance matching condition $Z_{C}=\sqrt{Z_{0} / G}$ is $T=1 /\left(\omega_{0} \sqrt{Z_{0} G}\right)$. Here, we characterize the response of our matching circuit in both the frequency domain and time domain.
We do this by first modeling the reflectance of our resonant circuit. Combining Eqs. (A1) and (A3), the reflectance as a function of frequency can be written:

$$
\Gamma \approx \frac{2\left(\frac{\omega}{\omega_{0}}-1\right)-i\left(Q_{\mathrm{int}}^{-1}-Q_{\mathrm{ext}}^{-1}\right)}{2\left(\frac{\omega}{\omega_{0}}-1\right)-i\left(Q_{\mathrm{int}}^{-1}+Q_{\mathrm{ext}}^{-1}\right)} .
$$

We fit this expression to the measured reflection as a function of frequency, as shown in Figs. 5(a) and 5(b) to extract $\omega_{0}, Q_{\text {int }}$, and $Q_{\text {ext }}$. As the SET is tuned into and out of Coulomb blockade its conductance varies significantly, which is reflected in $Q_{\text {int }}$ varying by as much as $75 \%$ over the range of an entire Coulomb peak in Fig. 5(c). In contrast, the external quality factor is constant, as it does not depend on the SET conductance. As a result, the total $Q$ varies from 19 to 42 across a single Coulomb peak. The full width, half maximum bandwidth of the circuit is $11.7 \mathrm{MHz}$ when the SET conductance is maximum, and $5.3 \mathrm{MHz}$ in Coulomb blockade. It is the parasitic capacitance $G_{0}$ that determines $Q_{\text {int }}$, hence our results in Coulomb blockade, where $Q_{\text {int }}=200$, implies $G_{0}=2.5 \mu \mathrm{S}$. If $G_{0}$ were to go to 0 , this would give a factor of 1.12 improvement in $\left|\Delta V_{\text {out }}\right|$.
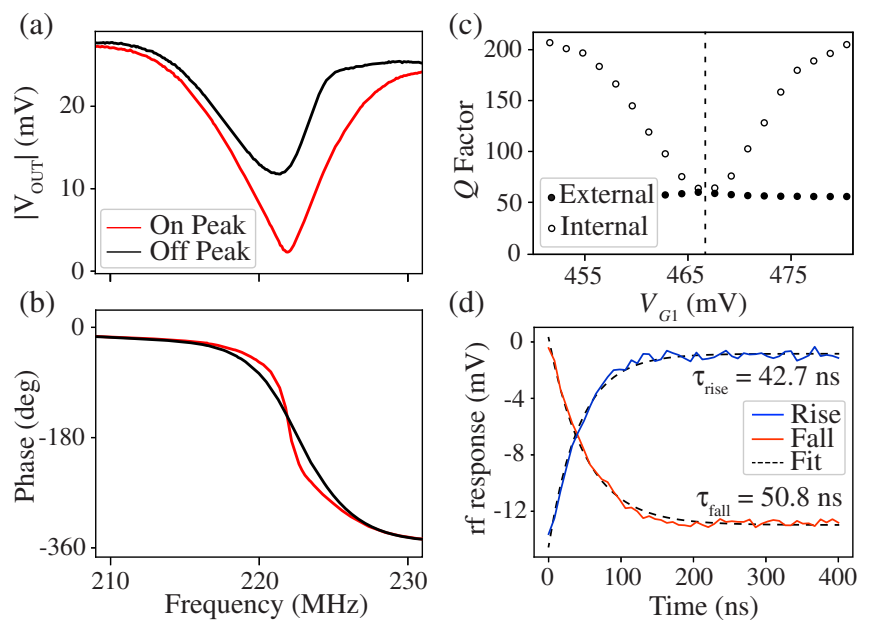

FIG. 5. Characterization of the resonator circuit. Panels (a) and (b) show the rf amplitude $\left|V_{\text {OUT }}\right|$ and phase, respectively, as a function of the rf-input frequency used showing a clear resonance around $223 \mathrm{MHz}$. The resonance changes significantly between cases in (black) and out (red) of Coulomb blockade. (c) Internal (empty circles) and external (filled circles) quality factors as a function of gate voltage across a Coulomb peak. The external quality factor is constant while the internal quality factor changes by a factor of 4 . Dotted lines show when internal and external quality factors are approximately equal, corresponding to the impedance matching condition. (d) Rising (blue) and falling (red) response of the resonating circuit as it is pulsed in and out of Coulomb blockade by a $50 \mathrm{kHz}$ square pulse on a nearby electrical control gate. The responses are fitted to exponential decays (black dash) to extract a rise time $T_{\text {rise }}=42.7 \mathrm{~ns}$ and fall time $T_{\text {fall }}=50.8 \mathrm{~ns}$, reflecting the change in $Q$. 
A time domain characterization of the circuit response is shown in Fig. 5(d). Here we apply a square voltage wave to gate $G 2$ to alternate between maximum and minimum SET conductance. To calculate the response time from this data we take time cuts through the square pulse edges of the rf response and fit them to an exponential function to extract the characteristic response time. Because the total quality factor of the circuit is different for the voltage position of the two cases, fits to the rise (blue) and fall (red) of the signal give different response times: a rise time $T_{\text {rise }}=42.7 \mathrm{~ns}$ and fall time $T_{\text {fall }}=50.8 \mathrm{~ns}$.

To extract a rise time via $Q$-factor analysis we must consider the relationship between bandwidth and rise time, $T_{\text {rise }}=2 Q / \omega_{0}$. We then substitute in the on-peak $Q$ factor $Q_{\max }=18.6$, which results in a rise time $T_{\text {rise }}=26.5 \mathrm{~ns}$, and on-peak $Q$ factor $Q_{\min }=42.4$, which results in the fall time $T_{\text {fall }}=60.5 \mathrm{~ns}$. This particular derivation method can be less reliable than the exponential pulse fits as it depends on the accuracy of fitting our resonating circuit model to the data. Based on the previous response of the circuit to the square voltage wave we determine the slowest response time to be $51 \mathrm{~ns}$. It is this response time which limits the minimum readout time in our experiment. The response time can be improved by an order of magnitude by a similar increase in the working frequency $\omega_{0}$.

\section{APPENDIX C: REACHING THE SHOT-NOISE LIMIT}

The noise in an ideal SET readout experiment will be fundamentally limited by the shot noise [17]. To be able to see the shot noise, and other limiting noise, in our setup we perform a measurement of the noise power spectral density output from our amplifier chain (before demodulation) measured at $222.3 \mathrm{MHz}$, with no rf input applied, as a function of gate voltage $V_{G 1}$ shown in Fig. 6(b). We apply a source bias up to $V_{S}= \pm 10 \mathrm{mV}$, larger than practical for detection, to reach the regime in which shot noise dominates [41]. At low bias, $\left|V_{S}\right|<3 \mathrm{mV}$, the dominant noise source is from the preamplifier (CMT CITLF-3). Here, the Coulomb diamond structure can still be observed, because in Coulomb blockade the impedance seen by the preamplifier input is mismatched from $50 \Omega$ and the noise level increases [41]. The noise level is minimum at Coulomb peaks or bias points where the total impedance of the tank circuit and SET is close to $50 \Omega$. At higher bias, the noise level increases approximately linearly with bias, consistent with shot noise of the SET, which has power spectral density of current fluctuations $S_{I I}=2 e F I$ [42]. After accounting for the impedance matching circuit, this contributes $S_{I I}=(2 e F I) Z_{\mathrm{SET}} / Z_{0}$ to the input of the preamplifier. The linear dependence of this noise on current allows us to fit the total gain of our measurement chain to be $77.1 \mathrm{~dB}$ (assuming $F=1$ at high bias) [41]. Using this gain factor we scale the measured noise to a noise temperature
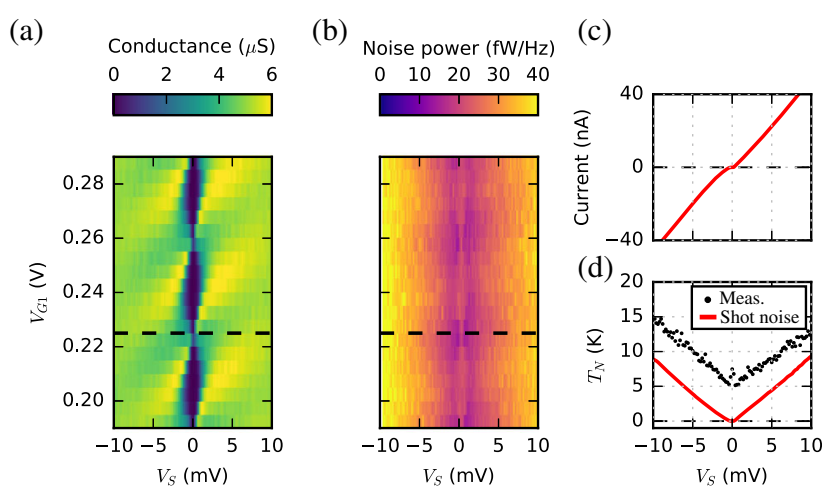

FIG. 6. Measured noise levels. (a) dc conductance of the SET as a function of dc bias $V_{S}$ and gate voltage $V_{G 1}$ showing the Coulomb diamond structure. (b) Simultaneously measured power spectral density output from the rf measurement chain at 222.3 MHz as a function of $V_{S}$ and $V_{G 1}$. At low bias, noise from the preamplifier dominates and is modulated by the conductance of the SET. At high bias, the noise level is linear with the current in the SET, consistent with SET shot noise. (c) dc current through the SET as a function of $V_{S}$ at the black dashed line in (a) and (b) $\left(V_{G 1}=0.225 \mathrm{~V}\right)$. (d) Measured noise output at the dashed line in (b), scaled to an equivalent noise temperature referred to the input of the preamplifier (black). The estimated shot-noise contribution to this noise is plotted as the red curve.

referred to the input of the preamplifier, shown in black dots in Fig. 6(d). The red curve indicates the shot-noise part of the total measured noise. The minimum noise temperature contributed by the preamplifier is $5.2 \mathrm{~K}$ (which is again higher in Coulomb blockade). We note that for the optimum input power used to perform spin readout, $-95 \mathrm{dBm}$, the SET rms current is about $1.8 \mathrm{nA}$, which has a corresponding shot-noise temperature $1.0 \mathrm{~K}$. Thus the preamplifier is the dominant noise source in our detection measurements, and if this noise could be reduced to the shot-noise limit we would achieve more than a factor of 5 increase in the voltage SNR. Adding an isolator between the matching circuit and the preamplifier would also improve the sensitivity in this experiment, as the impedance at the input of the preamplifier would remain $50 \Omega$ even when the SET is in Coulomb blockade.

\section{APPENDIX D: SENSITIVITY THEORY}

The shot-noise limited charge detection sensitivity of the strong-response SET has been addressed theoretically by Oxtoby et al. [17]. The minimum timescale for charge detection is the same as the timescale for electrons to transit the SET channel in the high conductivity SET state $\left(\sim I_{1} / e\right)$. At this timescale the uncertainty in tunneling events cannot be treated as Gaussian white noise, and how this noise combines with Gaussian white noise [such as from a preamplifier as considered in Fig. 2(c) in the main text] is complex. However, for simplicity we model the shot noise as Gaussian white noise with a current power spectral 
density $S_{I I}=2 e F I$, where $F$ is the Fano factor [42]. We assume that the SET is biased so that the current through the SET is 0 for one particular charge state and $I_{\mathrm{ON}}$ for the other. The current delivered to the transmission line in the ON state, assuming perfect impedance matching, is $I_{1}=I_{\mathrm{ON}} \sqrt{Z_{\mathrm{SET}} / Z_{0}}$. The shot noise, illustrated as a current source in Fig. 4, contributes noise power $2 e I_{\mathrm{ON}} Z_{\mathrm{SET}}$ to the output of the matching circuit, again assuming perfect impedance matching. We assume additional (white, Gaussian) current noise of spectral density $4 k_{B} T_{N} / Z_{0}$ is added to the output current by an amplifier chain of noise temperature $T_{N}$. Then the current noise variances are

$$
\begin{gathered}
\sigma_{0}^{2}=\frac{4 k_{B} T_{N}}{Z_{0} \tau}, \\
\sigma_{1}^{2}=\frac{4 k_{B} T_{N}}{Z_{0} \tau}+\frac{2 e F I_{\mathrm{ON}}}{\tau} \frac{Z_{\mathrm{SET}}}{Z_{0}} .
\end{gathered}
$$

And the measurement time $\tau_{m}$ such that $\left|I_{1}-I_{0}\right| /$ $\sqrt{\left(\sigma_{0}^{2}+\sigma_{1}^{2}\right) / 2}=2$ is

$$
\tau_{m}=\frac{2}{I_{\mathrm{ON}}^{2} Z_{\mathrm{SET}}}\left(8 k_{B} T_{N}+2 e F I_{\mathrm{ON}} Z_{\mathrm{SET}}\right) .
$$

To calculate the curves shown in Fig. 2(c) of the main text, we used $F=1$ and $Z_{\mathrm{SET}}=100 \mathrm{k} \Omega$, similar to the present experiment. In the limit of no added amplifier noise, $T_{N} \rightarrow 0$, the shot-noise limited measurement time is $\tau_{m} \approx 4 e F / I_{\mathrm{ON}}$. This is approximately twice the average time it takes for one electron to transit the SET. Future theoretical work will investigate the absolute limit of charge detection sensitivity for an ideal value of $Z_{\mathrm{SET}}$.

\section{APPENDIX E: ESTIMATED OPTIMAL READOUT TIME IN THE SHOT-NOISE LIMIT}

We can predict the optimal performance of single-shot readout in a precisely placed donor device in silicon operated in the strong-response regime at the shot-noise limit using guidelines presented by Keith et al. [32]. The guidelines consist of a set of conditions required to achieve spin readout with measurement fidelity $>99 \%$. The most relevant of these conditions include the following.

(i) The spin relaxation time $T_{1}$ is much greater than electron tunneling times, i.e.,

$$
T_{1} \gg t_{\mathrm{IN}}^{1}, t_{\mathrm{OUT}}^{1}, t_{\mathrm{IN}}^{0}, t_{\mathrm{OUT}}^{0},
$$

where $t_{\mathrm{IN}}^{1}\left(t_{\mathrm{IN}}^{0}\right)$ and $t_{\mathrm{OUT}}^{1}\left(t_{\mathrm{OUT}}^{0}\right)$ are the characteristic times for a spin-up (spin-down) electron to tunnel on and off the donor dot, respectively. (ii) Thermal excitations of spin-down electrons are sufficiently low, i.e.,

$$
t_{\text {OUT }}^{0} \gtrsim 800 t_{\text {OUT }}^{1}
$$

(iii) The sample rate $\Gamma_{s}$ (inverse of integration time) is fast compared to the length of the high signal response ("blip") during readout $\left(t_{\mathrm{IN}}^{0}\right)$, i.e.,

$$
\Gamma_{s} \gtrsim 12 / t_{\mathrm{IN}}^{0} \text {. }
$$

(iv) Signal-to-noise ratio is greater than 3 .

The optimal readout time $t_{\mathrm{opt}}$ is given in terms of $T_{1}$ and characteristic tunnel times as

$$
t_{\mathrm{opt}}=\frac{T_{1} t_{\mathrm{OUT}}^{0} t_{\mathrm{OUT}}^{1} \ln \left(\frac{t_{\mathrm{OUT}}^{0}\left(T_{1}+t_{\mathrm{OUT}}^{1}\right)}{T_{1} t_{\mathrm{OUT}}^{1}}\right)}{T_{1}\left(t_{\mathrm{OUT}}^{0}-t_{\mathrm{OUT}}^{1}\right)+t_{\mathrm{OUT}}^{0} t_{\mathrm{OUT}}^{1}} .
$$

By substituting each of the above conditions into the equation for $t_{\mathrm{opt}}$, as well as choosing a readout chemical potential such that $t_{\mathrm{OUT}}^{1}=t_{\mathrm{IN}}^{0}$, we find that $t_{\mathrm{opt}} \gtrsim 6.6 t_{\mathrm{IN}}^{0}$; i.e., the optimal readout time is approximately 7 times the characteristic blip length. To get $S N R \gtrsim 3$ (i.e., achieve noise a factor of $2 / 3$ lower) would require increasing the integration time to $450 \mathrm{ps}$ (assuming white Gaussian noise). This translates to an optimal $t_{\mathrm{IN}}^{0} \gtrsim 5.4 \mathrm{~ns}$ and, hence, $t_{\mathrm{opt}} \gtrsim 36 \mathrm{~ns}$.

Practically, with current sensing methods, it would be very difficult to achieve a response time close to $450 \mathrm{ps}$ given it is 2 orders of magnitude faster than in this paper. For comparison, an impedance-matched donor-based rf SET with center frequency of $5 \mathrm{GHz}$ could achieve a bandwidth $\sim 100 \mathrm{MHz}$ and sample rate $\sim 5 \mathrm{~ns}$. Using the shot-noise limit sensitivity of 200 ps in combination with the $5 \mathrm{~ns}$ sample rate results in an optimal readout time of $\sim 400 \mathrm{~ns}$. The SNR in this case would be much greater than 3 (SNR 10), in order to reach the requirement for 99\% readout fidelity, considering that the readout would be limited by the response time rather than the sensitivity, similar to the experiment presented here.

\section{APPENDIX F: DETERMINATION OF SPIN READOUT MEASUREMENT FIDELITY}

Single-shot electron spin readout can be performed in a global magnetic field via a process known as spin-to-charge conversion (STC) [33]. Pulsed gate voltages are used to load the donor dot with a random electron spin state, read the spin state via energy selective tunneling, and empty the donor dot ready to repeat. During the read phase the SET chemical potential is aligned between the two spin energy levels so that only $|\uparrow\rangle$ has enough energy to tunnel off the donor dot to then be replaced by a $|\downarrow\rangle$ electron. The pair of tunnel events ideally only occur when the initial electron state is $|\uparrow\rangle$. The resultant charge movements are detected 
by the rf SET as a sharp switch back and forth between two distinct rf-response levels $\left(R_{0}\right.$ and $\left.R_{1}\right)$. Examples of readout traces for both $|\uparrow\rangle$ (blue) and $|\downarrow\rangle$ (red) are shown in the main text [Fig. 3(a)]. Each readout trace is determined to be either initially $|\uparrow\rangle$ or $|\downarrow\rangle$, in a process called electrical readout, by comparing the maximum if SET response within the readout time $\Delta t$ of the readout trace to a particular threshold.

The quality of spin readout is quantified by the average percentage, known as the measurement fidelity $F_{M}$ of initial spin states that are correctly identified. The measurement fidelity is separated into state fidelities $\left(F_{\uparrow}\right.$ and $F_{\downarrow}$ ) based on initial spin state. Each of the state fidelities can further be broken down into STC fidelities which take tunneling, thermal, and relaxation errors into account, and electrical readout fidelities which accounts for signal noise, sampling, and filtering errors. The STC (electrical) state fidelities $F_{\text {STC }}^{\uparrow}$ and $F_{\text {STC }}^{\downarrow}\left(F_{E}^{\uparrow}\right.$ and $\left.F_{E}^{\downarrow}\right)$ for $|\uparrow\rangle$ and $|\downarrow\rangle$, respectively, are combined and optimized via the visibility $V_{\mathrm{STC}}=F_{\mathrm{STC}}^{\uparrow}+F_{\mathrm{STC}}^{\downarrow}-1\left(V_{E}=F_{E}^{\uparrow}+F_{E}^{\downarrow}-1\right)$. We then define $F_{M}$ as

$$
F_{M}=\frac{F^{\uparrow}+F^{\downarrow}}{2}
$$

where the overall state fidelities $F^{\uparrow}$ and $F^{\downarrow}$ are

$$
\begin{aligned}
& F^{\uparrow}=F_{\text {STC }}^{\uparrow} F_{E}^{\uparrow}+\left(1-F_{\text {STC }}^{\uparrow}\right)\left(1-F_{E}^{\downarrow}\right), \\
& F^{\downarrow}=F_{\text {STC }}^{\downarrow} F_{E}^{\downarrow}+\left(1-F_{\text {STC }}^{\downarrow}\right)\left(1-F_{E}^{\uparrow}\right) .
\end{aligned}
$$

The STC fidelities and visibility are shown as a function of $\Delta t$ in Fig. 3(c) of the main text while ignoring errors from other sources such that $F_{\mathrm{STC}}^{\uparrow}=F^{\uparrow}\left(F_{\mathrm{STC}}^{\downarrow}=F^{\downarrow}\right)$. This relation is determined from a series of tunnel rate equations,

$$
\begin{aligned}
F_{\mathrm{STC}}^{\uparrow}= & \frac{1}{\tilde{T}}\left[\tilde{T}-\tau_{\mathrm{OFF}}^{\uparrow} \tau_{\mathrm{OFF}}^{\downarrow} \exp \left(\Delta t / \tau_{\mathrm{OFF}}^{\downarrow}\right)\right. \\
& -T_{1} \tau_{\mathrm{OFF}}^{\downarrow} \exp \left(-\Delta t / T_{1}-\Delta t / \tau_{\mathrm{OFF}}^{\uparrow}\right) \\
& \left.+T_{1} \tau_{\mathrm{OFF}}^{\uparrow} \exp \left(-\Delta t / T_{1}-\Delta t / \tau_{\mathrm{OFF}}^{\uparrow}\right)\right], \\
F_{\mathrm{STC}}^{\downarrow}= & \exp \left(-\Delta t / \tau_{\mathrm{OFF}}^{\downarrow}\right),
\end{aligned}
$$

where $\tau_{\mathrm{OFF}}^{\uparrow}$ and $\tau_{\mathrm{OFF}}^{\uparrow}$ are the electron tunnel rates off the donor dot, $T_{1}=20 \mathrm{~s}$ is the spin-lattice relaxation time [23], and $\tilde{T}=T_{1} \tau_{\mathrm{OFF}}^{\downarrow}-T_{1} \tau_{\mathrm{OFF}}^{\uparrow}+\tau_{\mathrm{OFF}}^{\downarrow} \tau_{\mathrm{OFF}}^{\uparrow}$ for compactness. To maximize fidelity the readout duration needs to be long enough to capture all $|\uparrow\rangle$ tunnel events without giving enough time for thermal excitations to allow $|\downarrow\rangle$ electrons to tunnel or for $|\uparrow\rangle$ electrons to decay before tunneling. The optimal $\Delta t=1.5 \mu$ s leads to a STC visibility of $V_{\mathrm{STC}}=97.8 \%$.

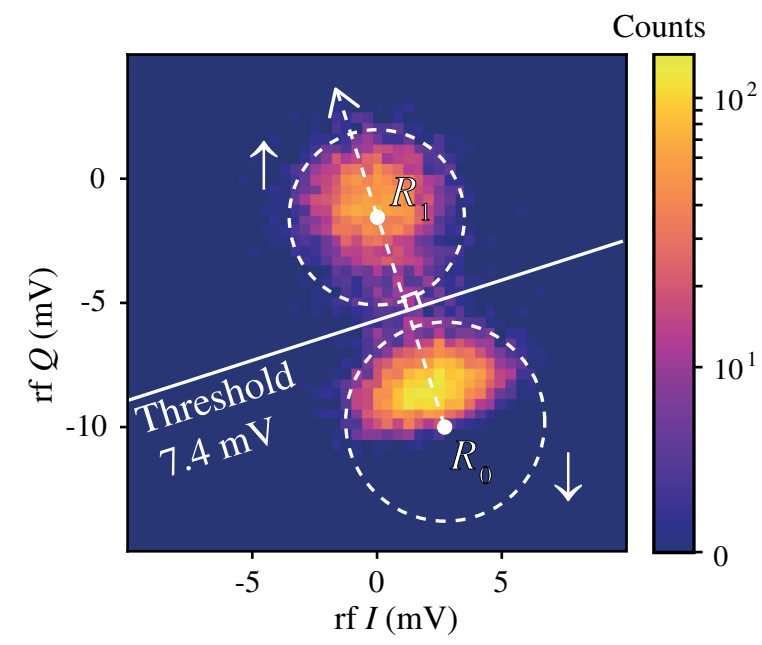

FIG. 7. $I-Q$ histogram of the maximum readout traces' responses. A 2D histogram of the maximum response of 10000 readout traces was constructed in $I-Q$ space. The areas within the two white, dotted circles centered around $R_{0}$ and $R_{1}$ depict the distributions of the entire traces that the maximum signals had been sampled from. The histogram shows two distinct distributions corresponding to spin-up and -down that can be separated by a perpendicular threshold $7.4 \mathrm{mV}$ from $R_{0}$ to distinguish the spin states during electrical readout.

Independent of STC conversion, electrical readout involves distinguishing the two possible spin states by applying a threshold to the measured SET response. Any traces that rise above the threshold within $\Delta t$ are declared $|\uparrow\rangle$ and, conversely, traces that do not rise above the threshold are declared $|\downarrow\rangle$. A histogram of 10000 readout traces in $I-Q$ space that shows two distinct peaks (with respective means $R_{0}$ and $R_{1}$ and standard deviations $\sigma_{0}$ and $\sigma_{1}$ ) corresponding to the two possible outcomes is shown in Fig. 7. The simplest threshold in this $2 \mathrm{D}$ space is a line perpendicular to the line joining $R_{0}$ and $R_{1}$, with the threshold being depicted by the solid line in Fig. 7 with a perpendicular distance of $7.4 \mathrm{mV}$ from $R_{0}$. The SNR of our data is high enough to permit collapsing the issue into the classic 1D case based on the projection of each point onto the line joining $R_{0}$ and $R_{1}$. The maximum response is defined as the point in $I-Q$ space furthest from $R_{0}$, in the direction of $R_{1}$, when projected along the common axis that joins the two points $R_{0}$ and $R_{1}$ as depicted by the dotted arrow in Fig. 7. The complement of the state fidelities $\left(F^{\uparrow}\right.$ and $F^{\downarrow}$ ) and visibility $V=F^{\uparrow}+F^{\downarrow}-1$ are shown in Fig. 8 with respect to the threshold used to distinguish tunnel events. The fidelities $F^{\uparrow}$ and $F^{\downarrow}$ are used to quantify the percentage of correctly identified $|\uparrow\rangle$ and $|\downarrow\rangle$ electrons, respectively. In Fig. 8 we calculate the state fidelities without accounting for heating or decoherence errors to independently characterize the electrical detection and, hence, $F_{E}^{\uparrow}=F^{\uparrow}\left(F_{E}^{\downarrow}=F^{\downarrow}\right)$. The fidelity $F^{\downarrow}$ does not reach above $90 \%$ until the threshold is $\sim 5 \mathrm{mV}$, where it 


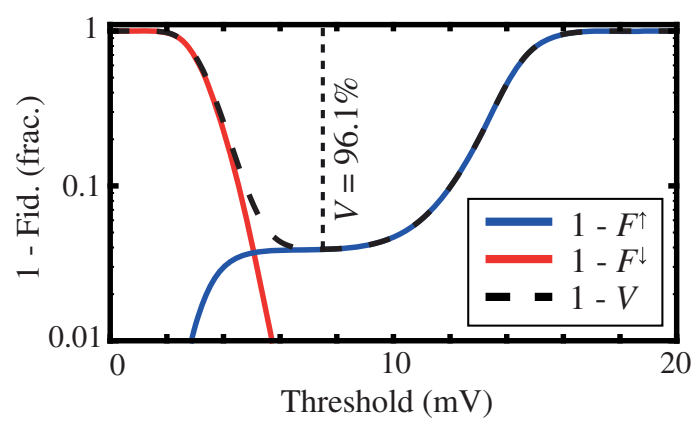

FIG. 8. Electrical readout fidelity. The complement of the electrical fidelities $F_{\text {STC }}^{\uparrow}$ (blue) and $F_{\text {STC }}^{\downarrow}$ (red) are shown as a function of the threshold used to distinguish between the two spin states. Reduction in fidelity is either caused by electrical noise or missing very fast tunnel events due to filtering or the limitation of the resonating circuit response time. The optimal threshold in terms of the visibility $V_{\mathrm{STC}}=F_{\mathrm{STC}}^{\uparrow}+F_{\mathrm{STC}}^{\downarrow}-1$ is found to be $7.4 \mathrm{mV}$ from $R_{0}$ resulting in $V=96.1 \%$.

falls below the noise floor of $R_{0}$, but by this point $F^{\uparrow}$ has already dropped sharply from missing short tunnel events that are filtered out by the resonator's 51 ns response time and fail to reach $R_{1}$. At the optimal threshold $V_{E}=96.1 \%$, which when combined with $V_{\text {STC }}$ gives an overall measurement fidelity $F_{M}=97 \%$.

[1] D. Loss and D. P. DiVincenzo, Quantum Computation with Quantum Dots, Phys. Rev. A 57, 120 (1998).

[2] D. S. Wang, A. G. Fowler, and L. C. L. Hollenberg, Surface Code Quantum Computing with Error Rates Over 1\%, Phys. Rev. A 83, 020302(R) (2011).

[3] Z.-L. Xiang, S. Ashhab, J. Q. You, and F. Nori, Hybrid Quantum Circuits: Superconducting Circuits Interacting with Other Quantum Systems, Rev. Mod. Phys. 85, 623 (2013).

[4] E. Kawakami, P. Scarlino, D. R. Ward, F. R. Braakman, D. E. Savage, M. G. Lagally, M. Friesen, S. N. Coppersmith, M. A. Eriksson, and L.M.K. Vandersypen, Electrical Control of a Long-Lived Spin Qubit in a Si/SiGe Quantum Dot, Nat. Nanotechnol. 9, 666 (2014).

[5] M. Veldhorst, C. H. Yang, J. C. C. Hwang, W. Huang, J. P. Dehollian, J. T. Muhonen, S. Simmons, A. Laucht, F. E. Hudson, H. M. Itoh, A. Morello, and A. S. Dzurak, A TwoQubit Logic Gate in Silicon, Nature (London) 526, 410 (2015).

[6] T. F. Watson, S. G. J. Philips, E. Kawakami, D. R. Ward, P. Scarlino, M. Veldhorst, D. E. Savage, M. G. Lagally, Mark Friesen, S. N. Coppersmith, M. A. Eriksson, and L. M. K. Vandersypen, A Programmable Two-Qubit Quantum Processor in Silicon, Nature (London) 555, 633 (2018).

[7] D. M. Zajac, A. J. Sigillito, M. Russ, F. Borjans, J. M. Taylor, G. Burkard, and J. R. Petta, Resonantly Driven CNOT Gate for Electron Spins, Science 359, 439 (2018).

[8] A. G. Fowler, M. Mariantoni, J. M. Martinis, and A. N. Cleland, Surface Codes: Towards Practical Large-Scale Quantum Computation, Phys. Rev. A 86, 032324 (2012).
[9] C. D. Hill, E. Peretz, S. J. Hile, M. G. House, M. Fuechsle, S. Rogge, M. Y. Simmons, and L.C. L. Hollenberg, A Surface Code Quantum Computer in Silicon, Sci. Adv. 1, e1500707 (2015).

[10] D. Risté, M. Dukalski, C. A. Watson, G. de Lange, M. J. Tiggelman, Ya. M. Blanter, K. W. Lehnert, R. N. Schouten, and L. DiCarlo, Deterministic Entanglement of Superconducting Qubits by Parity Measurement and Feedback, Nature (London) 502, 350 (2013).

[11] K. W. Murch, S. J. Weber, C. Macklin, and I. Siddiqi, Observing Single Quantum Trajectories of a Superconducting Quantum Bit, Nature (London) 502, 211 (2013).

[12] A. Morello, J. J. Pla, F. A. Zwanenburg, K. W. Chan, K. Y. Tan, H. Huebl, M. Mottonen, C. D. Nugroho, C. Yang, J. A. van Donkelaar, A. D. C. Alves, D. N. Jamieson, C. C. Escott, L. C. L. Hollenberg, R. G. Clark, and A. S. Dzurak, Single-Shot Readout of an Electron Spin in Silicon, Nature (London) 467, 687 (2010).

[13] T. F. Watson, B. Weber, M. G. House, H. Büch, and M. Y. Simmons, High-Fidelity Rapid Initialization and Read-Out of an Electron Spin via the Single Donor $D^{-}$Charge State, Phys. Rev. Lett. 115, 166806 (2015).

[14] A. M. Tyryshkin, S. Tojo, J. J. L. Morton, H. Riemann, N. V. Abrosimov, P. Becker, H.-J. Pohl, T. Schenkel, M. L.W. Thewalt, K. M. Itoh, and S. A. Lyon, Electron Spin Exceeding Seconds in High-Purity Silicon, Nature (London) 11, 143 (2012).

[15] M. Steger, K. Saeedi, M. L. W. Thewalt, J. J. L. Morton, H. Riemann, N. V. Abrosimov, P. Becker, and H.-J. Pohl, Quantum Information Storage for over 180 s Using Donor Spins in a ${ }^{28} \mathrm{Si}$ "Semiconductor Vacuum", Science 336, 1280 (2012).

[16] J. T. Muhonen, J. P. Dehollain, A. Laucht, F. E. Hudson, R. Kalra, T. Sekiguchi, K. M. Itoh, D. N. Jamieson, J. C. McCallum, A. S. Dzurak, and A. Morello, Storing Quantum Information for 30 Seconds in a Nanoelectronic Device, Nat. Nanotechnol. 9, 986 (2014).

[17] N. P. Oxtoby, H. M. Wiseman, and H.-B. Sun, Sensitivity and Back Action in Charge Qubit Measurements by a Strongly Coupled Single-Electron Transistor, Phys. Rev. B 74, 045328 (2006).

[18] A. N. Korotkov and M. A. Paalanen, Charge Sensitivity of the Radio Frequency Single-Electron Transistor, Appl. Phys. Lett. 74, 4052 (1999).

[19] V. O. Turin and A. N. Korotkov, Analysis of the RadioFrequency Single-Electron Transistor with Large Quality Factor, Appl. Phys. Lett. 83, 2898 (2003).

[20] V. O. Turin and A. N. Korotkov, Numerical Analysis of Radio-Frequency Single-Electron Transistor Operation, Phys. Rev. B 69, 195310 (2004).

[21] H. Brenning, S. Kafanov, T. Duty, S. Kubatkin, and P. Delsing, An Ultrasensitive Radio-Frequency SingleElectron Transistor Working Up to $4.2 \mathrm{~K}$, J. Appl. Phys. 100, 114321 (2006).

[22] W. W. Xue, Z. Ji, F. Pan, J. Stettenheim, M. P. Blencowe, and A. J. Rimberg, Measurement of Quantum Noise in a Single-Electron Transistor Near the Quantum Limit, Nat. Phys. 5, 660 (2009).

[23] T F. Watson, B. Weber, Yu-L. Hsueh, L. C. L. Hollenberg, R. Rahman, and M. Y. Simmons, Atomically Engineered 
Electron Spin Lifetimes of $30 \mathrm{~s}$ in Silicon, Sci. Adv. 3, e1602811 (2017).

[24] M. H. Devoret and R. J. Schoelkopf, Amplifying Quantum Signals with the Single-Electron Transistor, Nature (London) 406, 1039 (2000).

[25] D. M. Zajac, T. M. Hazard, X. Mi, E. Nielsen, and F. R. Petta, Scalable Gate Architecture for a One-Dimensional Array of Semiconductor Spin Qubits., Phys. Rev. Applied 6, 054013 (2016).

[26] Y. Makhlin, G. Schon, and A. Shnirman, Quantum-State Engineering with Josephson-Junction Devices, Rev. Mod. Phys. 73, 357 (2001).

[27] A. Shnirman and G. Schon, Quantum Measurements Performed with a Single-Electron Transistor, Phys. Rev. B 57, 15400 (1998).

[28] C. Barthel, Control and Fast Measurement of Spin Qubits, Ph.D. thesis, MIT, 2010.

[29] M. G. House, I. Bartlett, P. Pakkiam, M. Koch, E. Peretz, J. van der Heijden, T. Kobayashi, S. Rogge, and M. Y. Simmons, High-Sensitivity Charge Detection with a SingleLead Quantum Dot for Scalable Quantum Computation, Phys. Rev. Applied 6, 044016 (2016).

[30] L. A. Tracy, D. R. Luhman, S. M. Carr, N. C. Bishop, G. A. Ten Eyck, T. Pluym, J. R. Wendt, M. P. Lilly, and M. S. Carroll, Single Shot Spin Readout Using a Cryogenic HighElectron-Mobility Transistor Amplifier at Sub-Kelvin Temperatures, Appl. Phys. Lett. 108, 063101 (2016).

[31] A. Fuhrer, M. Füchsle, T. C. G. Reusch, B. Weber, and M. Y. Simmons, Atomic-Scale, All Epitaxial In-Plane Gated Donor Quantum Dot in Silicon, Nano Lett. 9, 707 (2009).

[32] D. Keith, S. K. Gorman, L. Kranz, Y. He, J. G. Keizer, M. A. Broome, and M. Y. Simmons, Benchmarking High Fidelity Single-Shot Readout of Semiconductor Qubits, New J. Phys. 21, 063011 (2019).

[33] J. M. Elzerman, R. Hanson, L. H. W. van Beveren, B. Witkamp, L. M. K. Vandersypen, and L. P. Kouwenhoven,
Single-Shot Read-Out of an Individual Electron Spin in a Quantum Dot, Nature (London) 430, 431 (2004).

[34] M. A. Broome, T. F. Watson, D. Keith, S. K. Gorman, M. G. House, J. G. Keizer, S. J. Hile, W. Baker, and M. Y. Simmons, High-Fidelity Single-Shot Singlet-Triplet Readout of Precision-Placed Donors in Silicon, Phys. Rev. Lett. 119, 046802 (2017).

[35] J. Stehlik, Y.-Y. Liu, C. M. Quintana, C. Eichler, T. R. Hartke, and J. R. Petta, Fast Charge Sensing of a CavityCoupled Double Quantum Dot Using a Josephson Parametric Amplifier, Phys. Rev. Applied 4, 014018 (2015).

[36] M. Koch, J. G. Keizer, P. Pakkiam, D. Keith, M. G. House, E. Peretz, and M. Y. Simmons, Spin Read-Out in Atomic Qubits in an All-Epitaxial Three-Dimensional Transistor, Nat. Nanotechnol. 14, 137 (2019).

[37] B. D'Anjou and W. A. Coish, Optimal Post-Processing for a Generic Single-Shot Qubit Readout, Phys. Rev. A 89, 012313 (2014).

[38] D. M. Pozar, Microwave Engineering (John Wiley and Sons, Inc., New York, 2004).

[39] S. Hellmuller, M. Pikulski, T. Muller, B. Kung, G. PueblaHellmann, A. Wallraff, M. Beck, K. Ensslin, and T. Ihn, Optimization of Sample-Chip Design for Stub-Matched Radio-Frequency Reflectometry Measurements, Appl. Phys. Lett. 101, 042112 (2012).

[40] G. Puebla-Hellmann and A. Wallraff, Realization of Gigahertz-Frequency Impedance Matching Circuits for Nano-Scale Devices, Appl. Phys. Lett. 101, 053108 (2012).

[41] A. Aassime, G. Johansson, G. Wendin, R. J. Schoelkopf, and P. Delsing, Radio-Frequency Single-Electron Transistor as Readout Device for Qubits: Charge Sensitivity and Backaction, Phys. Rev. Lett. 86, 3376 (2001).

[42] S. Kafanov and P. Delsing, Measurement of the Shot Noise in a Single-Electron Transistor, Phys. Rev. B 80, 155320 (2009). 